\title{
Knowledge of Farmers about Pest Management Practices in Pambaimadu, Vavuniya District: An Ordered Probit Model Approach
}

\author{
Subashiny Nagenthirarajah ${ }^{1}$ and S. Thiruchelvam ${ }^{2}$ \\ IPostgraduate Institute of Agriculture, University of Peradeniya \\ ${ }^{2}$ Department of Agricultural Economics and Business Management, \\ Faculty of Agriculture University of Peradeniya, Sri Lanka. \\ sthiru@pdn.ac.lk
}

\begin{abstract}
Similar to many developing countries, pesticide related issues in Sri Lanka have become a major concern in the recent past. Many researches were conducted to evolve technical alternatives for eco-friendly agriculture, but very less emphasis was given to the knowledge and behaviour of the farmers. Having recognized the need, present study was conducted to assess the farmers' knowledge level on pest management practices and socio-economic factors influencing the existing pest management practices. Ordered Probit model was used to analyze the data from 50 vegetable farmers who were randomly selected from Pampaimadu, Agricultural Instructor range in the Vavuniya district. Nearly $60 \%$ of the farmers had medium level of knowledge of plant protection practices. Only $6 \%$ of the farmers had good level of knowledge towards the recommended plant protection measures. Almost all the farmers depended on chemical pesticides for the management of pest and diseases but at $35 \%$ higher concentration than recommended level. Farming experience and social participation were positive and significantly contributed to farmers' knowledge on safe pesticide use. Education, income, age and family size of farmers had showed no relationship with the knowledge level of pest management. Increased social participation and, effective extension programmes are important to improve farmers' knowledge on proper use of pesticides and its effect on their health and environment.
\end{abstract}

Key Words: Farmers Pest Management Knowledge, Ordered Probit Model,

\section{Introduction}

Pesticides use is a double-edged sword in the battle against pests as it affects human health and also contaminates the environment. Misuse and Overuse of pesticide is very common among farmers of developing economies and Sri Lanka is no exception. Sri Lankan farmers have a tendency to ignore technical recommendations and base usage on their own experience often leading to indiscriminate application (Wilson and Tiddsell, 2001). Yet, some farmers have a genuine problem of insufficient knowledge and information on the and spraying pesticides on their farms are exposed to pesticides and suffer from numerous morbidity effects ranging from headaches, nausea to cramps 
and body tremors. According to World Health Organization (WHO) in 2005 the estimated occupational pesticides poisoning affected as many as 25 millions of the agricultural work force each year in developing countries. Acute pesticide poisoning has become a major problem in Sri Lanka. Farmers handling and spraying pesticides using hand sprayers suffer from numerous morbidity effects (Sivayoganathan et al., 1995).

Human health effects due to routine occupational exposures in pesticide application have not been well documented in Sri Lanka but assumed that continuous use of pesticides has caused human ill health and agricultural sustainability significantly. Wilson and Tiddsell (2001) have estimated the private cost of farmers' exposure to pesticides in Sri Lanka. They found that a farmer on average incurs a cost of around Rs 5465 a year (equal to about a month income) due to exposure to pesticides. On the other hand, use of the avertive /defensive behavior approach estimates the cost to be around Rs 405 a year or $12 \%$ of the monthly income of an average farmer per year. The contingent valuation method estimates give a higher figure of Rs 11,471 or cost of more than $2 \mathrm{l} / 2$ months income a year due to ill health resulting from exposure to pesticides. The intangible and indirect costs such as discomfort, stress, pain and suffering are important costs that are associated with exposure to pesticides. Deaths from exposure to pesticides are not uncommon. Hospital statistics in Sri Lanka show that on average 14,500 individuals were admitted to government hospitals and around 1500 individuals a year died from pesticide poisoning during the period 1986-1996 (National Poison Information Centre, 1997). The damage done from exposure to pesticides, not only to human health, but also to the native fauna and the environment in general is very large. The effect on neighboring individuals is likely to be considerable since water sources and the entire environment are affected. The entire food chain can be affected as a result. The damage done to consumers of cultivated food crops, though unknown, could also be high. Over use of pesticides occur due to lack of knowledge about environmental ill effects.

Although acute pesticide poisoning occurs at alarmingly high rates in Sri Lanka, it is a major problem through out the developing world. The worldwide incidence is about three million cases and 220,000 deaths each year. The largest numbers are in developing countries. Each year tens of thousands of farmers, especially in developing countries, are affected by exposure to pesticides. According to recent estimates 3 million people are poisoned and 200,000 died by pesticide poisoning each year (FAO, 2005). In Sri Lanka pesticide poisoning causes many deaths, particularly among males $40-54$ years and the elderly. The suicide rate in Sri Lanka is about 160 per 100,000. Apart from these hospital data, various field studies carried out have also confirmed high levels of morbidity from direct exposure to pesticides ranging 
from faintish feelings, headaches, nausea, diarrheoa, muscle twitching, rashes and cramps (Sivayoganathan et al., 1995).

Vegetable crop sector in Vavuniya district accounts for heavy use of pesticides. Pesticides are extensively used on upland cash crops due to higher susceptibility to pest and diseases and relatively higher economic returns from these crops. Usually, liquid formulation and wet-table powders are applied as spray mixture while granule and powders are broadcast simply by hand. Nowadays, increased attention is being paid on to selection of suitable pesticides, its formulation method and judicious use of pesticides to minimize risk and detrimental effects on health and environment. Department of Agriculture, Vavuniya has been promoting integrated approach for managing pest problems (IPM) in crop production from the last decade (Administration Report, 2003). In the long term, the wide spread adoption of IPM will be the only way to satisfy public demand for safe and sustainable pesticide usage. IPM is practiced in rice cultivation but very little on crops such as chillie and onion. In general insecticides are heavily used in the vegetable sector followed by the fungicides. Pesticide use continued to remain the most popular method of pest control by farmers even though pesticide showed harmful effects on human health, environment and crop ecosystem (Administrative Report, 2005).

With the background discussed above, present study was conducted to assess the farmers' knowledge level on pest management practices and socioeconomic factors influencing the existing pest management practices and farmers' awareness about the ill-effects of pesticides use on the farmers' health and the environment.

\section{Methodology}

\section{Study Area}

Vavuniya District in the Northern Province of Sri Lanka was selected for this study because of the following reasons. Nearly 28,000 ha of land are being cultivated with paddy and other major field crops such as chillie, onion and vegetables. Lift irrigation from wells is utilized for the subsidiary food crops like chille, red onions, big onions and grain legumes, exotic and local vegetables. Annually, an average of 2145ha of land is used to cultivate vegetables and produce $34,575 \mathrm{mt}$ of vegetable during maha and yala season in which $50 \%$ is contributed by the yala season (Administration Report, 2005). The study area selected for the study was Bambaimadu Agricultural instructor (AI) range, which is a typical agricultural area that is threatened by high pesticide use in the Vavuniya district. Fifty vegetable farmers who cultivate chiille, red onion and vegetables in more than 0.25 ha were randomly 
selected from three selected villages. Secondary data was extracted from the reports of the Department of Agriculture and base hospital, Vavuniya.

Keeping in view the objective of the study, socio-economic variables like farmers' age, education, family size, occupation, and experience in farming, operational holding, annual income, extension contacts and social participation were collected as independent variables. Farmers' knowledge on pesticides use practices and their awareness about the ill effects of pesticides on their health and environment were computed as dependent variable. Knowledge of farmers on cultural, mechanical and chemical protection practices and their ill effects of pesticides use were computed based on a simple five-point Lickert scale method. Knowledge scales were composed of approximately equal numbers of favourable and unfavourable statements concerning the pesticide use in vegetable cultivation, environment and health. Farmers were asked to respond to each statement in terms of their level of agreement. Scale scores were computed by summing the response scores of the component items with the response given, using the following integral values: Strongly agree 5, agree 4, undecided 3, disagree 2, or strongly disagree 1 . The mean and standard deviations of measurements were used to categorize the respondents' knowledge of pesticides use (KNOW) into three groups viz., low, medium and high level of knowledge. The general form to identify farmers' knowledge of pesticide use is as follows

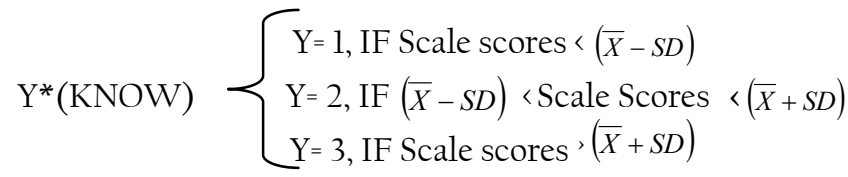

Where

$\bar{X}$ - Mean of knowledge and awareness of pesticide use,

$S D$ - standard deviation

To determine the factors that influence perceived ill effects of pesticide use, the above-calculated dependent variable was then regressed on the set of socio economic variables, using the Ordered Probit Model described below.

\section{Ordered Probit Model}

Response chosen in an opinion survey often appears as a discrete choice set rather than a continuous one. When the dependent variable takes more than two values, but these values have a natural ordering, as is common in survey responses, the ordered probit model is often appropriate. The Ordered Probit model is a fairly straightforward extension of the binary probit model that 
can be used in cases where there is multiple and ranked discrete dependent variables. Discrete choice variables fall into two categories: The first one is ordered variables and the second one is unordered variables. The previous studies have mostly utilized multiple regression models and binary probit or log it models to study statistical relations between dependent and explanatory variables. Because of the discrete nature of the dependent variable in this study, ordinary least squares regression would be an inappropriate model. Probit or logit model specification is utilized for dichotomous dependent variables and it gives discrete outcomes too. With this in mind, multinomial model for discrete choice of ordered data is more applicable for this data analysis. Nevertheless the ordered probit model, which can make full use of every response choice, is statistically more efficient than the binary logit and probit model. Therefore the follwing ordered probit model that uses Maximum Likelihood (ML) analysis (Borooah, 2002) was employed in this study. Models for ordinal dependent variables can be formulated as a threshold model with a latent dependent variable:

$$
Y^{*}=\beta^{!} X+\epsilon
$$

Where, $\mathrm{Y}^{*}$, is unobserved. It is assumed that is normally distributed with zero mean. , is a vector of unknown parameters to be estimated. X, is a vector of a respondent characteristics.

Equation (l) can be used to specify the empirical model given in equation (2).

$\mathrm{Y}^{*}=\beta_{0}+\beta_{1} F S I Z E+\beta_{2} E D U+\beta_{3} E M P+\beta_{4} E X P+\beta_{5} L A N D+\beta_{6} A I N C+\beta_{7} S O C+\varepsilon$

Where

\begin{tabular}{|c|c|c|}
\hline Variable Name & Description of Variable & Type of Variable \\
\hline \multirow[t]{2}{*}{ KNOW } & Ordered Dependent Variable & $\mathrm{Y}^{*} 1$ to 3 levels \\
\hline & $\begin{array}{l}\text { Farmers' knowledge about safe } \\
\text { pesticide use and its ill effect } \\
\text { on the Environment and health }\end{array}$ & Ordinal \\
\hline FSIZE & $\begin{array}{l}\text { Number of member of the } \\
\text { farmers' family }\end{array}$ & Discrete \\
\hline AGE & Farmers' Age & Continuous \\
\hline
\end{tabular}


Number of years of farmers' education Discrete

EMP

Full time farming -1, Part time farming-0 Dummy

AINC

Net annual household income

Continuous

EXP

No of years of experience in farming

Continuous

$\mathrm{SOC}$

Social participating score

Dummy

\section{Results and discussion}

\section{Profile of vegetable cultivating farmers}

Analysis of the data collected on socio-economic variables of chillie and red onion cultivators in Pampaimadu Vavuniya district indicated that farmers had an average age of 47 years with average number of experience in farming of 16 years (Table 1). The average family size was around 4 per family, which is lower than the national average of family size of 4.3. Formal education was average with almost $60 \%$ of the farmers having up to only llyears of schooling. About $21 \%$ had less than primary education. Agriculture training was low in the area as only $25 \%$ of farmers attended an agriculture-training programme conducted by the Department of Agriculture. The average of operational landholding was 0.81 ha. The yearly average income of the household was Rs. 161,160 . The average nature of farming was 0.78 , which explains that most of the farmers were full time vegetable farmers. At least $22 \%$ of the farmers had additional sources of income, which demanded approximately $10 \%$ of their working time and contributed approximately $15 \%$ of total family income. The average social participation score was 0.82 , which indicates that most of the farmers were members of social organizations and participating in some form of social activities in the village.

Table l: Personal traits of vegetable farmers - Pampaimadu, Vavuniya Distrcit

\begin{tabular}{|l|c|c|c|c|c|}
\hline Variable & Obs. & Mean & Std. Dev. & Min. & Max. \\
\hline AWAR (Scale) & 50 & 25.44 & 7.1860 & 12 & 40 \\
\hline FSIZE (No.) & 50 & 4.02 & 1.8460 & 1 & 7 \\
\hline AGE (Yrs.) & 50 & 46.52 & 11.8274 & 27 & 73 \\
\hline EDU (Yrs.) & 50 & 10.96 & 2.2943 & 5 & 18 \\
\hline EMP (Scale) & 50 & 0.78 & 1.5556 & 0 & 3 \\
\hline AINC (Rs.) & 50 & 161160 & 80324.94 & 36000 & 368000 \\
\hline LAND (ha.) & 50 & 0.81 & 4.2162 & 0.2024 & 1.822 \\
\hline EXP (Yrs.) & 50 & 16.16 & 6.8254 & 4 & 35 \\
\hline SOC (Scale) & 50 & 0.82 & 0.3881 & 0 & 1 \\
\hline
\end{tabular}




\section{Knowledge on safety uses of pesticide}

At present insecticides are heavily $(70 \%)$ used in the vegetable sector folowed by fungicides (20\%). Weedicide use was very minimal in this sector $(10 \%)$, except in onion. It was also found that the majority of the pesticides were newly introduced and highly toxic chemicals. No one was concerned about long-term ill effects and consequence of pesticide use on human's health and environment. Almost all the farmers depended on chemical peticides for the management of pest and diseases and most of them use moerately toxic chemicals but at 35\% higher concentration than recommended level. Farmers' choice of pesticide was primarily based on efficacy rather than safety.

In the study area, $80 \%$ of the respondents were over mixing than using the recommended rate (Table 2 ). Some of them were using wrong mixtures in chemical use such as combining use of Marshal and Lannet. The reasons for over mixing were that recommended rates were not effective due to resurgence of pest to chemicals. Farmers were having low knowledge about pesticide use. Significant percentage (78\%) of the farmers was concerned about the chemicals expiry date. About $72 \%$ of the farmers undertook the spraying themselves due to the reason that carelessness of labourers cost more for them in social and economical point of view. About $48 \%$ of the hired laborers did not consider wind direction while applying chemicals. Some of the farmers were also spraying in the evening because they were working in other sectors. But most of the hired labor was spraying in the morning and evening. These statistics show that most of the farmers were having medium knowledge about general knowledge of handling.

Table 2: The status knowledge on handling Pesticides

\begin{tabular}{|c|c|c|}
\hline General Knowledge of handling & No. of farmers & $\%$ \\
\hline Mixing ratio (over) & 40 & $80 \%$ \\
\hline Concern on Expiry date & 39 & $78 \%$ \\
\hline Concern on Wind direction & 24 & $48 \%$ \\
\hline Family labour & 36 & $72 \%$ \\
\hline Hired Labour & 14 & $28 \%$ \\
\hline
\end{tabular}

Farmers did not adopt (Table 3) precautionary measures using fully body covers such as mask, gloves and caps when using all chemicals. Some of the farmers were saying that usually we cover our body when using harmful pesticides. Betel chewers and smokers almost totally avoided these habits during spraying. Farmers were in opinion that present chemicals were in less potent persuading them to over use chemicals. However, among the various pesticides used by farmers, nearly $50 \%$ of the brand used in the 
study area falls under WHO categories of class I and class II, which were more frequently $(70 \%)$ used by farmers compared to other pesticides. In the study area, $60 \%$ of the farmers take a bath with soap after spraying pestcides, indicating their concern on precautionary measures. These statistics show that most of the farmers were having medium knowledge about prcautionary measures while handling and spraying pesticides.

Table 3: Knowledge on precautionary measures (When spraying harmful pesticide)

\begin{tabular}{|l|c|c|}
\hline Practicing precautionary measures & No. of farmers & $\%$ \\
\hline Wearing mask and gloves & 5 & $10 \%$ \\
\hline Wearing mask and wearing caps & 8 & $16 \%$ \\
\hline Wearing mask, gloves and caps & 0 & $0 \%$ \\
\hline Avoid chewing Betel & 49 & $98 \%$ \\
\hline Avoid smoking tobacco & 50 & $100 \%$ \\
\hline $\begin{array}{l}\text { Cleaning body with soap before } \\
\text { taking food }\end{array}$ & 30 & $60 \%$ \\
\hline
\end{tabular}

Table 4 highlights that farmers were highly negligent in the proper disposal of empty containers. It was also observed that many small boutiques display the large size empty pesticide cans. In all, the knowledge on correct disposal method implied a high knowledge gap for farmer. It is recommended that burying is the safest method, but very few farmers (14\%) had adopted this practice others threw them either on field or dumped in bush areas adjoining their field. About 22\% of the farmers reuse and sell the empty bottles and large size cans to store kerosene, other oil items and used small size bottles to make oil lamp. About $16 \%$ of the farmers were burning empty plastic bottles. This shows that most of the farmers were having low-level knowledge about disposal and storage methods of pesticide.

Table 4: Knowledge of disposal and storage method

\begin{tabular}{|l|c|c|}
\hline Method of disposal & No. of Farmers & $\%$ \\
\hline Re using and selling & 11 & $22 \%$ \\
\hline Burying & 07 & $14 \%$ \\
\hline Throwing on land & 24 & $48 \%$ \\
\hline Burning & 08 & $16 \%$ \\
\hline Store in boundary of house & 30 & $60 \%$ \\
\hline
\end{tabular}

\section{Knowledge and awareness about ill effects of pesticides}

Nearly $60 \%$ of the farmers had medium level of knowledge of plant protetion practices. The average score of farmers' awareness about ill effects of 
pesticide on humans' health and the environment was 25.44. This implies that most of the farmers were having medium level awareness. Most farmers preferred to prevent insect pest rather than control. Therefore, sprayed peticides to crops whether there was insects or pest to eliminate potential risk. Only $6 \%$ of the farmers had good level of knowledge towards the recomended plant protection measures and understanding of long-term ill effects of pesticides on their health and environment. The IPM strategy for vegetables and other food crops were not available.

\section{Ordered probit regression analysis}

The results of the probit analysis of the 50 observations are presented in Table 5. The goodness of fit of the model show reasonably high pseudo $\mathrm{R}^{2}$ of 0.55 and significant at $1 \%$ level, which suggest that $54 \%$ of the variability in knowledge can be explained by the seven selected socio economic variables in the ordered probit regression model.

The ordered probit model focused on factors that influence the farmers' knowledge about the use and ill effects of pesticide on the environment and health. Farming experience and social participation were positive and significantly contributed to farmers' knowledge on the ill effects of pesticide use on humans' health and the environment. Education, incomes, age and family size of farmers had showed no relationship with the knowledge level of pest management. Social participation depends on existing institutional structures, particularly on mutual communication and information. It does not rely on the level of education, age and income.

Table 5. Factor influencing farmers' knowledge and use of pesticides

$\begin{array}{llll}\text { Log likelihood } & =-20.877862 & \text { Number of obs. }=50 & \text { LR chi2 }(7)=50.94 \\ \text { Prob }>\text { chi2 } & =0.0000 & \text { Pseudo R2 } & =0.5495\end{array}$

\begin{tabular}{|l|r|c|c|c|c|c|}
\hline & Coef. & Std. Err & $z$ & $P>z$ & $95 \%$ & Confidence Interva \\
\hline FSIZE & -.0710 & .1469 & -0.48 & 0.629 & -.3588 & .2169 \\
\hline EDU & -.0325 & .1351 & -0.24 & 0.810 & -.2972 & .2323 \\
\hline EMP & -.0125 & .1809 & -0.07 & 0.945 & -.3670 & .3420 \\
\hline AINC & $2.31 \mathrm{e}-06$ & $3.57 \mathrm{e}-06$ & 0.65 & 0.518 & $-4.69 \mathrm{e}-0$ & $9.31 \mathrm{e}-06$ \\
\hline EXP & $.19651^{* *}$ & .0550 & 1.57 & 0.000 & .0888 & .3042 \\
\hline LAND & -.05577 & .2752 & -0.20 & 0.839 & -.5952 & .4836 \\
\hline SOC & $2.4313^{* * *}$ & .7557 & 3.22 & 0.001 & .9502 & 3.9125 \\
\hline
\end{tabular}

Significant Level: ** $\mathrm{P}<0.05$ and $* * * \mathrm{P}<01$ 
Correlation analysis shows higher positive correlation of $\mathrm{r}=0.834$ of farmers' farming experience with farmers knowledge about ill effects of pesticide on the environment and health. Furthermore, those who were participating more in social activities were having high awareness about ill effects of pesticides on the environment and health. Farmers' social participation had positive significant correlation of $\mathrm{r}=0.614$ with farmers' knowledge about ill effects of pesticide on the environment and heath. The family size, nature of farming, annual income and operational land holding were insignificant and further farmers' annual income and farmers' education had negative correlation with farmers' awareness on ill effects of pesticide uses. However, experience and social participation, which reflect some ethical considerations borne by the people, provide some hope for safe use of chemicals.

\section{Conclusion}

The appraisal of the knowledge status of the vegetable cultivating farmers towards pest management indicated that almost all the farmers were dependent on chemical pesticides for the management of insect pest and diseases and most of them were using moderately toxic pesticides but at a very high frequency and dosage. About $60 \%$ of the vegetable-cultivating farmers were having a medium level of awareness and only $6 \%$ of the farmers were having a above average level about the pesticide use and their ill effect. Lack of knowledge on the ill effects of agrochemicals made them to over use chemicals and affects their health, sustainability of agriculture and the environment. Ordered Probit model results indicated that farming experience and social participation were positive and significantly contributed to farmers' knowledge and awareness of the ill effects of pesticide use on humans' health and the environment.

Increased social participation and, effective extension programmes are important to improve farmers' knowledge on proper use of pesticides and its effect on their health and environment. Agricultural extension need to be employed to follow a systematic, well planned and coordinated approach in the area for improving the knowledge status of vegetable growers towards pest management. In this regard there is a need for biological and social scientists to collaborate more closely to help farmers make better decisions regarding pesticide use.

\section{References}

Administration Report(2003 and 2005) Department of Agriculture, Vavuniya.

Borooah, V.K. (2002) Logit and Probit: Ordered and Multinomial Models. Series: Quantitative Applications in the Social Sciences, No. 138, Thousand Oaks: Sage Publications. 
Food and Agricultural Organization, (FAO), (2005) FAOSTAT Database, www. apps.fao.org, Accessed April 10 2005.

National Poisons Information Centre, (1997) General Hospital Colombo, Sri Lanka, www. infolanka.com/people/shyam/clinMed7.htm, Accessed May $7^{\text {th }} 1997$.

Sivayoganathan, C., Gnanachandran, S., Lewis, J. and Fernando, M. (1995) Protective measure use and symptoms among agro pesticide, applicators in Sri Lanka', Social Science and Medicine, v. 40, pp. 431-436.

Wilson, C. and Tisdell, C. (2001) Why farmers continue to use pesticides despite environmental, health and Sustainability costs, Ecological Economics, v. 39, pp. 449-462.

World Health Organization, (WHO), (2005) www.joem.org/pt/re/joem, Accessed February $11^{\text {th }} 2005$. 